\title{
Lamotrigine serum levels: ceiling effect in people with epilepsy in remission?
}

Jennifer D’Anto ${ }^{1}$, Wlodzimierz Wnuk ${ }^{2}$, Andrea O. Rossetti ${ }^{3}$, Thierry Buclin ${ }^{4}$, Jan Novy 3

1Bachelor of Medicine, University of Lausanne, Switzerland. 'Laboratory of Lavigny, Lavigny, Switzerland. ${ }^{3}$ Neurology Service, Department of clinical neurosciences, Centre Hospitalier Universitaire Vaudois (CHUV) and University of Lausanne, Switzerland. ${ }^{4}$ Division of clinical pharmacology, Centre Hospitalier Universitaire Vaudois (CHUV) and University of Lausanne, Switzerland.

Abstract word count: 183

Manuscript word count: 1423

The manuscript includes one figure

Key words: Drug naïve, response to medication, therapeutic drug monitoring.

\section{Corresponding author:}

Dr Jan Novy

Service de neurologie

Département des neurosciences cliniques

BH07

CHUV

Rue du Bugnon 46

1011 Lausanne

Phone : ++41213141190

Fax : ++41213141290

Email : jan.novy@chuv.ch 


\section{Abstract}

\section{Background}

Antiepileptic drugs titration in epilepsy remains empirical. Since in practice seizure remission may be obtained with low doses, we aimed to determine if patients in remission have lower lamotrigine levels than those who continue experiencing seizures.

\section{Methods}

Retrospective analysis of the distribution of lamotrigine levels in people in remission and with ongoing seizures in a cohort. Remission was defined as the longest of either 3 times of the longest interseizure interval, or one year. Only through levels were considered.

\section{Results}

Between 2009 and 2014, we identified 93 adults, 10 were in remission. People in remission had significantly ( $\mathrm{p}=0.018$ ) lower serum levels (median $2.3 \mathrm{mg} / \mathrm{l}$, range: $0.7-8.2$ ) than those with ongoing seizures (median $5.4 \mathrm{mg} / \mathrm{l}$, range: 1.1 18.2). We did not find any patient in remission with levels higher than $8.2 \mathrm{mg} / \mathrm{l}$. Distribution of dosages also differed among the groups, but less significantly $(\mathrm{p}=0.03)$.

\section{Conclusion}

A correlation between lamotrigine serum levels and seizure response in term of remission appears to exist, and a ceiling level above which remission is unlikely is proposed. This could help to rationalise the titration of medication in drugnaïve patients with epilepsy. 


\section{Introduction}

Prescription and titration of antiepileptic drugs (AEDs) remains largely empirical, dosage being increased sometimes until the appearance of adverse events in patients with ongoing seizures. There is indeed no well-established AED ceiling dosage in term of seizure control. It has been however shown that more than $90 \%$ of seizure-free patients were taking less than $300 \mathrm{mg}$ of lamotrigine per day, suggesting that the chance to achieve seizure freedom at higher doses is likely to be small(1). Such ceiling dosage is particularly relevant in drug-naïve people with epilepsy, in whom the primary aim of the treatment is finding the right agent to achieve remission as quickly as possible, whereas in people with medically refractory epilepsy, continued titration resulting in a relative reduction of the seizure frequency can still be valuable.

Interindividual variability of AED metabolism due of genetic polymorphism or co-medication prevents establishing a direct correspondence of AED dosage with clinical response, which leads to the need of individualising AED dosage. This results in a stepwise increase in the search of the optimal dosage, the patient being potentially exposed to medication-related adverse events, and to continued seizures when maximal efficacious dosage of the medication would have been surpassed. Therapeutic drug monitoring offers the opportunity to determine to which AED concentration a patient is exposed, adjusting thus for the genetic variability of antiepileptic drugs metabolism or drug interactions.

The reference range of older-generation AEDs, such as phenytoin or carbamazepine, has been well studied, and drug level ranges are widely accepted. Older generation AEDs are however decreasingly used(2);(3). New generation AED, such as lamotrigine, have been shown to be more effective (thanks to their better tolerability) and lamotrigine became the standard of care in focal epilepsy (4).

Our aim was to explore the distribution of lamotrigine serum levels in a population of people with epilepsy stratified between patients in remission and with ongoing seizures, in order to confirm that the former have lower serum levels and to identify a ceiling level associated with remission. 


\section{Methods}

We reviewed retrospectively lamotrigine concentrations and the response to medication at the time of blood sampling. We collected all consecutive lamotrigine levels at our centre determined at the Laboratory of Lavigny between August 2009 and March 2014. We considered only the most recent dosage if the patient had more than one analysis. Lamotrigine serum levels were measured by high performance liquid chromatography.(5) We ascertained through chart reviews that all lamotrigine levels were measured at through concentration. Cases when the interval between the last dosing and the blood sampling was less than 6 hours(6) and patients younger than 17 years old (some epileptic syndromes in this age group have a natural evolution leading to remission) were excluded.

Through computerised medical records, we collected basic epidemiologic data including demographics and pregnancy status. Seizure history was collected from clinical reports, which in our institution are standardised with systematic reporting of the last seizure. We defined remission according to the International League Against Epilepsy (ILAE)(7) as follows: the longest of either three times the longest free seizure interval (for example a seizure every 6 months, implies a seizure-free interval of 18 months) or at least one year. This rule of three has been recently supported statistically(8). All seizure types were considered for assessment of remission, including also auras. In case of insufficient documentation about the nature of a suspected relapse of seizure, episodes were conservatively considered as having seizures. People with breakthrough seizures due to a provocative factor (e.g., abusive alcohol intake or drug omission) were not considered in remission. In case of remission on a polytherapy, we considered the remission to be associated with lamotrigine, only if this was the last drug introduced before achieving it; if a clear sequence could not be established, the case was excluded. 
Distributions of concentration/dosage according to remission or ongoing seizures were performed using Student T or Mann-Whitney U test as needed. Analyses were performed using SPSS 21 (IBM inc.).

\section{Results}

We collected 168 patients with epilepsy of whom 9 were excluded because of incomplete data and 46 because the sample was performed less than 6 hours after dosing, or it was not possible to determine at what time the sample was taken. We also excluded 20 paediatric patients. This left 93 patients for analysis.

There were 47 women (50.3\%), median age was 50 years old (ranged: 17 to 92). Overall, $45(48 \%)$ were on monotherapy. Ten patients $(10.8 \%)$ were in remission, all of them were on lamotrigine monotherapy; none of them were pregnant at the time of sample.

Median daily dosage was $250 \mathrm{mg}$ (ranged: 25 to $1200 \mathrm{mg}$ ). Median though blood levels varied were at $4.9 \mathrm{mg} / \mathrm{l}$ (range: 0.6 to $18.2 \mathrm{mg} / \mathrm{l}$ ). Serum levels in people in remission and those with ongoing seizures are shown in figure 1. Distributions of serum logs levels were significantly different $(\mathrm{p}=0.018$, Mann Whitney test) between people in remission (median 2.3, range: 0.7-8.2) and those with ongoing seizures (median 5.4, range: 1.1-18.2). Among those in remission, 9 out of 10 had levels lower than $6 \mathrm{mg} / \mathrm{l}$. There was also a significant difference $(\mathrm{p}=0.03$, Mann Whitney test) in the distribution of daily dosages (with a ceiling dose of $300 \mathrm{mg}$ for remission) when comparing people in remission (median $175 \mathrm{mg}$, range: 50 $300 \mathrm{mg}$ ) with those with ongoing seizures (median 300mg, range: 25-1200mg).

\section{Discussion}

This study suggests that people in remission have significantly lower lamotrigine serum levels and daily dosages than those with uncontrolled epilepsy. It also shows that there might be a ceiling effect in the concentration associated with seizure remission, as there was no patient in remission with blood levels beyond $8.2 \mathrm{mg} / \mathrm{l}$. 
These data shows a correlation between clinical effects and drug concentration in a different way that previously studied. A previous study investigated the relationship with treatment response in terms of relative decrease in seizure frequency(9), but did not find any correlation. Relative seizure frequency decrease is likely to be flawed by regression to the mean(10), and therefore represents an outcome that is not very robust. Conversely, using remission according the current ILAE recommendation(7) seems more likely to account for a tangible change in the course of the disease (8). Another study(11) did not take into account the time of the sampling, making likely that some levels were artificially high. We considered only residual levels for a better consistency using samples taken at least 6 hours after dosing; a period where the difference with through period is likely to be minimal(12).

Lower serum levels in people in remission could appear contra intuitive, as higher dosage may be felt to bring a more potent effect. This approach would however not take into account that some people, whatever the dose they are exposed to, will not respond to the treatment, and if they will respond, it is likely to be at low or moderate dose(1).

If the present findings are confirmed, a ceiling serum level associated with remission for patients taking lamotrigine could help to rationalise the titration of the medication in drug naïve people with epilepsy. Relying on oral dosage is likely to be biased by interindividual variability of bioavailability, patients' weight, and drug interactions (with hormonal contraceptives for instance). We found not surprisingly a lesser difference between the two groups when comparing dosages, the ceiling dosage associated with remission was $300 \mathrm{mg}$ which is in keeping with a previous report(1). A ceiling serum level would allow estimating the probability of remission with further increase(s) of medication. This could allow switching earlier to another medication if the probability of remission appears small; thus avoiding further increase most associated with little difference in term of seizure control but potentially exposing the patient to unnecessary adverse events. 
This study has limitations. Its retrospective nature implies that samples were not taken systemically, and it is likely that a proportion of people with epilepsy in remission may not have had serum levels, as there would have been no practical consequence. The possible natural remission of the disease has to be considered(13), making the treatment unnecessary, explaining therefore low level observed in some of the patients. Including patients with relapses associated with an attempt of weaning could have been a possibility to ascertain this. A prospective study decreasing progressively the dosage to ascertain the smallest sufficient dose would require a prohibitively long period of observation. This does however not alter the finding that people in remission had lower serum levels.

In conclusion, serum AEDs level could be useful when titrating medication in drug-naïve patient with epilepsy, preventing unnecessary increments and time losses, and thus potentially increasing the quality of care. 


\section{References}

1. Kwan P, Brodie MJ. Effectiveness of first antiepileptic drug. Epilepsia. 2001;42(10):1255-60.

2. Johannessen Landmark C, Fossmark H, Larsson PG, Rytter E, Johannessen SI. Prescription patterns of antiepileptic drugs in patients with epilepsy in a nation-wide population. Epilepsy Res. 2011;95(1-2):51-9.

3. Malerba A, Ciampa C, De Fazio S, Fattore C, Frassine B, La Neve A, et al. Patterns of prescription of antiepileptic drugs in patients with refractory epilepsy at tertiary referral centres in Italy. Epilepsy Res. 2010;91(23):273-82.

4. Marson AG, Al-Kharusi AM, Alwaidh M, Appleton R, Baker GA, Chadwick DW, et al. The SANAD study of effectiveness of carbamazepine, gabapentin, lamotrigine, oxcarbazepine, or topiramate for treatment of partial epilepsy: an unblinded randomised controlled trial. Lancet. 2007;369(9566):1000-15.

5. Wnuk W. Simultaneous determination of seventeen antiepileptic compounds in serum by isocratic reversed-phase liquid chromatography. Ther Drug Monit. 2001;23(4):478.

6. Johannessen SI, Tomson T. Pharmacokinetic variability of newer antiepileptic drugs: When is monitoring needed? Vol. 45, Clinical Pharmacokinetics. 2006. p. 1061-75.

7. Kwan P, Arzimanoglou A, Berg AT, Brodie MJ, Hauser WA, Mathern G, et al. Definition of drug resistant epilepsy: Consensus proposal by the ad hoc Task Force of the ILAE Commission on Therapeutic Strategies. Epilepsia. 2010;51(6):1069-77.

8. Brandon Westover M, Cormier J, Bianchi MT, Shafi M, Kilbride R, Cole AJ, et al. Revising the "rule of Three" for inferring seizure freedom. Epilepsia. 2012;53(2):368-76.

9. Bartoli A, Guerrini R, Belmonte A, Alessandri MG, Gatti G, Perucca E. The influence of dosage, age, and comedication on steady state plasma lamotrigine concentrations in epileptic children: a prospective study with preliminary assessment of correlations with clinical response. Ther Drug 
Monit [Internet]. 1997;19(3):252-60.

10. Spilker B, Segreti A. Validation of the phenomenon of regression of seizure frequency in epilepsy. Epilepsia. 1984;25(4):443-9.

11. Kilpatrick ES, Forrest G, Brodie MJ. Concentration-effect and concentration-toxicity relations with lamotrigine: A prospective study. Epilepsia. 1996;37(6):534-8.

12. Theis JGW, Sidhu J, Palmer J, Job S, Bullman J, Ascher J. Lack of pharmacokinetic interaction between oxcarbazepine and lamotrigine. Neuropsychopharmacology. 2005;30(12):2269-74.

13. von Podewils F, Lapp S, Wang ZI, Hartmann U, Herzer R, Kessler C, et al. Natural course and predictors of spontaneous seizure remission in idiopathic generalized epilepsy: 7-27 years of follow-up. Epilepsy Res [Internet]. Elsevier B.V.; 2014;108(7):1221-7. 